\title{
EDITORIAL
}

\section{Tensor and Hypergraph}

\section{Shmuel FRIEDLAND ${ }^{1}, \quad$ Liqun $\mathbf{Q I}^{2}, \quad$ Yimin WEI $^{3}, \quad$ Qingzhi YANG ${ }^{4}$}

1 Department of Mathematics, Statistics and Computer Science, University of Illinois at Chicago, Chicago, IL 60607-7045, USA

2 Department of Applied Mathematics, The Hong Kong Polytechnic University, Hong Kong, China

3 School of Mathematical Sciences, Fudan University and Shanghai Key Laboratory of Contemporary Applied Mathematics, Fudan University, Shanghai 200433, China

4 School of Mathematical Sciences and LPMC, Nankai University, Tianjin 300071, China

(c) Higher Education Press and Springer-Verlag GmbH Germany 2017

Higher-order tensors are natural extensions of matrices; matrices are order-2 tensors. However, higher-order tensors represent multilinear maps, which are nonlinear unlike matrices.

In recent years, with the rapid flow of massive data in this digital world, the demand for effective and efficient data-processing and data-managing algorithms finds its way in connection with the theory of tensors and hypergraphs.

This special section contains 9 articles. We feel so strongly that the fruitful works reflecting the interweaving across tensors and hypergraphs covering

structured tensors (H. Chen and Y. Wang, C. Xu and Y. Xu, and L. Ye and Z. Chen),

tensor decompositions (J. Wen et al.),

tensor spectrum (L. Chen et al.),

tensor generalized inverse (J. Ji and Y. Wei),

tensor optimizations (X. Zhang et al.), and

hypergraph (D. Chen et al. and X. Yuan et al.),

which makes not only mathematicians but also physicists, computer scientists, and engineers, aware that there is a new branch called Tensor and Hypergraph.

We would like to thank all the authors, the referees, the editor-in-chief of Frontiers of Mathematics in China, Prof. Kung-Ching Chang, and the managing editor Shannian Lu for their hard work on this special section.

\footnotetext{
Received September 25, 2017

E-mail: friedlan@uic.edu, liqun.qi@polyu.edu.hk, ymwei@fudan.edu.cn, qz-yang@nankai.edu.cn
} 